\title{
V3SPA: A Visual Analysis, Exploration, and Diffing Tool for SELinux and SEAndroid Security Policies
}

\author{
Robert Gove* \\ Invincea Labs
}

\begin{abstract}
SELinux policies have enormous potential to enforce granular security requirements, but the size and complexity of SELinux security policies make them challenging for security policy administrators to determine whether the implemented policy meets an organization's security requirements. To address the challenges in developing and maintaining SELinux security policies, this paper presents V3SPA (Verification, Validation and Visualization of Security Policy Abstractions). V3SPA is a tool that can import SELinux and Security Enhancements (SE) for Android source or binary policies and visualize them using two views: A policy explorer, and a policy differ. The policy explorer supports users in exploring a policy and understanding the relationships defined by the policy. The diffing view is designed to support differential policy analysis, showing the changes between two versions of a policy.

The main contributions of this paper are 1) the design of the policy explorer, and the design and novel usecase for the policy differ, 2) a report on system design considerations to enable the graph visualizations to scale up to visualizing policies with tens of thousands of nodes and edges, and 3) a survey of five SELinux and SE for Android policy developers and analysts. The results of the survey indicate a need for tools such as V3SPA to help policy workers understand the big picture of large, complex security policies.
\end{abstract}

\section{INTRODUCTION}

As system complexity has increased, security goals have become incomprehensible at the implementation and configuration levels of an operational environment. To secure modern enterprise systems, the ability for security professionals to define and visualize the overall security policies of the system is essential to understanding and verifying policy configurations. While some tools exist to implement the system security policies themselves, the complexity of operational systems clutter the ability of security engineers to map security goals to proper system configurations, thus leaving a high probability of security errors. Furthermore, as system complexity increases, so does the difficulty in assessing differential system modifications without requiring a ground up re-verification.

Many Linux distributions have adopted SELinux, including Fedora, Red Hat Enterprise Linux, CentOS, and Gentoo. A version of SELinux has also been a part of Android since version 4.3, meaning that $81.1 \%$ of the more than one billion active Android devices employ SELinux ${ }^{1}$. Considering the wide adoption of SELinux and Security Enhancements (SE) Android, there is a clear need for policy administrators to have effective tools to design, implement, and verify SELinux policies. (In this paper, I use "SELinux security policies" to refer to both SELinux and SE for Android security policies, unless stated otherwise.)

SELinux policies are challenging to understand and analyze due to their size and complexity. The sesearch utility shows that the 20141203 version of the Tresys reference policy ${ }^{2}$ (the policy that all Linux distributions use as a base) shows there are 94,420 allow

\footnotetext{
*robert.gove@invincea.com

${ }^{1}$ As of July, 2016 https://developer.android.com/about/dashboards/index.html

${ }^{2}$ https://github.com/TresysTechnology/refpolicy
}

rules. These rules express the relationships between 3,410 subjects, 4,037 objects, 77 permissions, and 231 object classes.

To address the challenges faced by security engineers and analysts, this paper presents $\mathrm{V} 3 \mathrm{SPA}^{3}$, an open source interactive visualization tool for analyzing and exploring SELinux and SE for Android security policies. V3SPA is designed to be a scalable system capable of visualizing all the allow rules of a policy simultaneously, while allowing users flexibility to selectively apply filters to see only the relevant components of the policy. V3SPA has two main visualizations: A policy explorer (Figure 1), and a policy differ (Figure 2). These visualizations are designed to explore a policy to better understand the rules and relationships in the policy, and to visually diff two versions of a policy and analyze the differences between them. I discuss V3SPA's design, and I describe several design decisions and performance optimizations that enable V3SPA to scale up to visualizing tens of thousands of nodes and edges.

To motivate the need for visual tools to explore and analyze SELinux policies, I discuss the results of a survey of five SELinux policy developers. I compare V3SPA to the tools they commonly use, showing that V3SPA enables analysis that is difficult and time consuming using other tools. I also show V3SPA's utility by demonstrating V3SPA's features while analyzing several SELinux and SE for Android security policies.

\section{SELINUX AND SE FOR ANDROID}

Traditionally, Linux operated under a discretionary access control (DAC) model, where the owner of an object (such as a file) could grant access to it (read, write, execute) at the owner's discretion. Security Enhanced Linux (SELinux) is an add-on for Linux that implements mandatory access control (MAC) based policies on top of the existing DAC model $[9,11]$. In MAC, access control is specified at the system level by a policy that is defined and controlled by the system security policy administrator (see Loscocco et al. for further discussion of MAC in secure operating systems [10]). SELinux implements MAC through fine-grained type enforcement rules in the security policy, where subject types (typically processes, also called domains) are granted permissions on object types (i.e. system resources such as files, sockets, shared memory, etc.) and object classes (the specific class of the object) [11].

In SELinux, subjects (i.e. processes) are not allowed permissions on any object (e.g. a socket or a file) until it is specifically permitted by a policy rule. These policy rules are known as allow rules. For example, the allow user_t bin_t : file read; allow rule says that the processes in the user_t domain are allowed to read the files of the bin_t type. SELinux security policies also include definitions for users, roles, and other information. This collection of rules is the portion of an SELinux security policy that V3SPA analyzes ${ }^{4}$.

SE for Android (Security Enhancements for Android) [19] adds a version of SELinux to the Android mobile operating system. Though SE for Android and SELinux policies are not exactly the

\footnotetext{
${ }^{3}$ https://github.com/invincealabs/V3SPA

${ }^{4}$ Although SELinux is more than just MAC type enforcement $[9,11]$, V3SPA is designed to analyze the type enforcement policy rules, so this paper focuses on that part of SELinux
} 


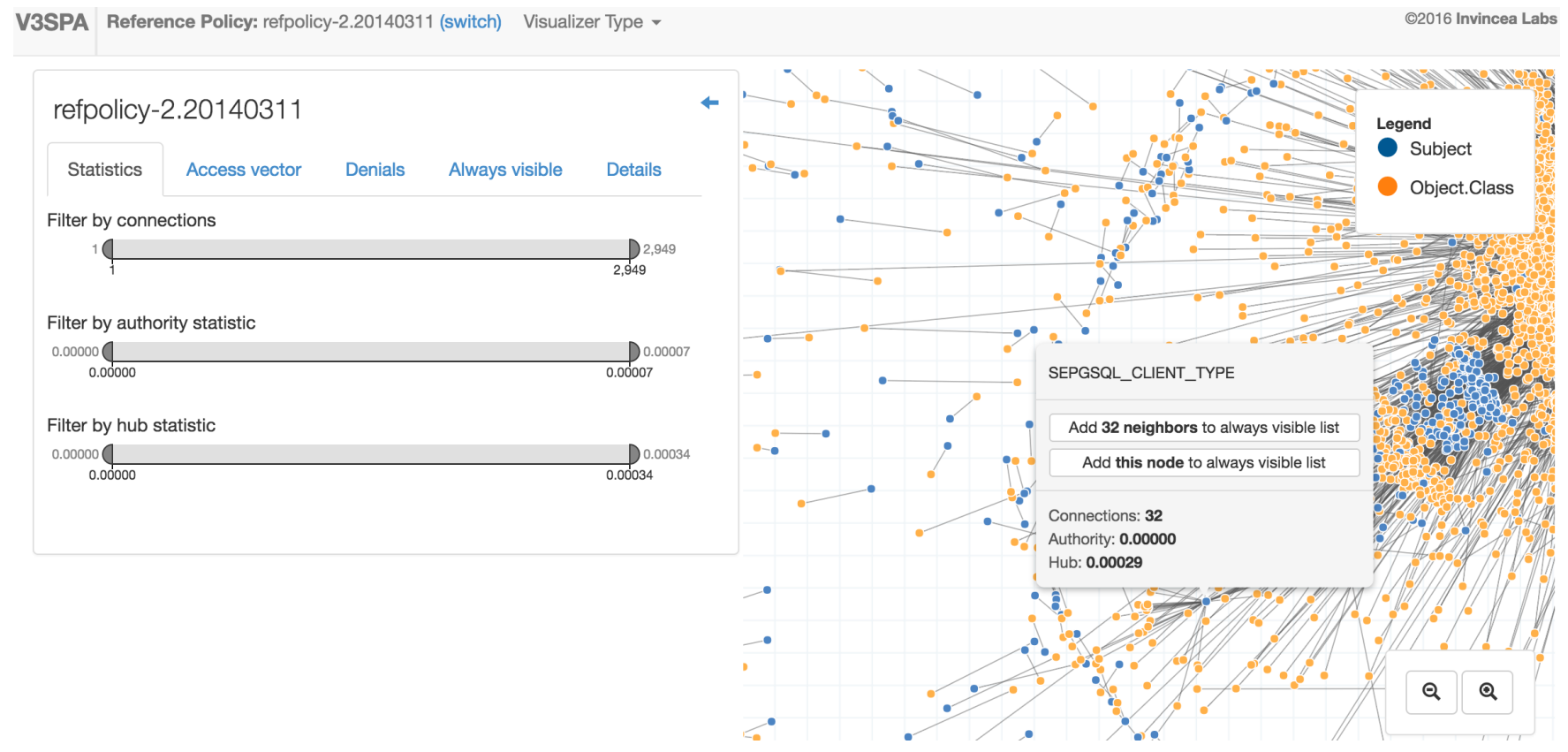

Figure 1: The policy explorer in V3SPA. On the left, the editor pane shows controls for filtering the policy. On the right is a node-link diagram representation of the policy, where subjects are blue nodes, and objects and classes are paired together into a single orange node if they both occur in the same allow rule. A tooltip for the sepgsql_client_type subject node shows statistics and filter options for the node.

same, they are similar enough that many tools can be used to develop and analyze both types of policies.

Policies begin as source that is then compiled into a policy binary that can be that can be installed and run on SELinux enabled systems.

For more information on SELinux, see Mayer et al. [13] and the original SELinux research $[9,10,11]$.

\section{Related Work}

Existing publicly available tools are commonly used in SELinux policy development and analysis. The Tresys SETools ${ }^{5}$ include several such command-line tools, such as sediff for comparing differences between two policies, sedta for performing domain transition analyses, seinfo for viewing the policy components, seinfoflow for analyzing information flow, and sesearch for searching the policy rules. SETools also includes apol, a tool with similar capabilities as the command-line tools, but with a graphical user interface. Policy developers commonly use text editors such as vi to write policy, and other command-line tools such as grep to search the policy. See Section 4 for a more detailed discussion of our survey on tools commonly used by policy developers. These tools can be useful, but due to the size and complexity of SELinux policies, they can be cumbersome for understanding the intricacies of even medium-sized policies. This is because the tools are primarily text based, which places a higher load on cognitive processes and working memory than well designed visualizations and graphical user interfaces.

Abstractly, we can consider SELinux security policies to be nodes (policy types) with links (policy permissions) expressing relationships between them. This is a common abstraction and can be seen in several research projects (e.g. [12, 23, 22, 15]).

NodeXL [20] and Gephi [2] are common tools for analyzing and exploring graph datasets. These tools can be very effective, but they are not designed to show the differences between two graphs, nor does their design take into account the extra semantics found

${ }^{5}$ https://github.com/TresysTechnology/setools in SELinux security policies, such as subject types having a set of permissions on objects and classes.

SEGrapher [12] is a tool designed specifically for visually analyzing SELinux policies. SEGrapher visualizes the policy as a node-link diagram, where nodes are types and links are policy rules connecting types. Because policies can be quite large and therefore challenging to analyze and visualize, SEGrapher employs a clustering algorithm to reduce the complexity of the visualization. The clustering algorithm clusters types based on a set of relations, and then allows users to see the clusters of object types that are accessed by user-selected subject types. This produces a smaller, simplified graph than showing all the types simultaneously. The downside is that users see an abstraction of the policy instead of the actual policy graph. SEGrapher also does not support comparing two versions of a policy.

Pan and $\mathrm{Xu}$ [15] proposed using treemaps and semantic substrates for visually analyzing multi-domain access control and cross-domain information exchange, a related but different usecase from MAC. Xu et al. [23, 22] also used semantic substrates, but in the specific context of SELinux security policies. They use semantic substrates to show users, roles, domains (subjects), and types (objects). Xu et al. supplemented semantic substrates with adjacency matrices to show paths between types. However, it is unclear whether these visualizations would scale to show large portions of a policy, and they were not designed to show the differences between two policies. To help analysts identify potential policy violations, they also implemented a visual query system intended to make it easier for typical system administrators to execute policy queries.

Chen et al. [3] extract attack graphs from SELinux and AppArmor policies, identify the minimal attack paths, and compare the Quality of Protection between different policies using the minimal attack paths. This approach is useful for identifying weaknesses in a system, but it is not designed to show all the differences between two versions of a policy.

Clemente et al. [4] propose a method to analyze logs of attacks on SELinux systems, and then visualize the information flows dur- 
ing attacks. The information flows are visualized as node-link diagrams, and edge color indicates the frequency of events occurring during attacks. These information flows can be useful, but their system requires access to system log files, which may not be available to policy designers and analysts. Their work was also not designed to show differences between policies.

Wang et al. [21] propose a semi-supervised machine learning approach to analyze SE for Android audit logs for the purpose of refining an existing policy. This is a worthwhile approach, but it does not help policy developers understand existing rules in a policy, and it is not designed to help analysts understand the differences between two policies.

Expandable Grids [17] is an interaction technique for visualizing and authoring security policies. It is designed to show the combined set of permissions that a subject will have on an object, and they allow users to expand the hierarchy to show more detail (e.g. to expand a directory to show permissions on individual files).

Outside of the context of security policies, TreeVersity [5] was designed to show differences between pairs of trees. In particular, TreeVersity shows differences in tree structure and numerical node attributes, but TreeVersity is not designed to scale to the thousands of nodes often present in SELinux security policies. TreeJuxtoposer [14] is designed to be able to compare structural changes between trees with hundreds of thousands of nodes. However, SELinux security policies do not have a strong sense of hierarchy that would lend themselves to tree visualization, and policy graph nodes, such as types or classes, do not typically have numerical attributes that would change between policies.

Visualization of temporal network evolution, AKA dynamic networks, is used to compare changes in the structure or attributes of a graph over time. There are many powerful techniques for dynamic network visualization (see Bach et al. [1] for a survey of several such techniques), but in general they are not designed to adequately capture changes in the unique semantics found in SELinux policy graphs. More concretely, dynamic network visualization tends to focus on showing added and removed nodes, added or removed edges, and changes in node or edge attributes, often over multiple timesteps. For policy graph comparison, users only need to compare two versions of a policy, and users need to understand the different kinds of nodes that were added or removed, information that is not typically shown in dynamic network visualizations.

V3SPA uses a grid and grouped layout for the policy differ. At present, there is active research in the area of grid and orthogonal graph layouts (e.g. Yoghourdjian et al. [24] and Kieffer et al. [7]). Although there could be advantages to the grid layouts proposed in the literature, such as reduced edge crossings, those approaches often require tens to hundreds of seconds to compute layouts. On the other hand, V3SPA's policy differ computes node layouts by simply sorting nodes by their policy, enabling real-time graph rendering. The grouped layout in the policy differ is similar to other grouped layouts, such as group-in-a-box [18], but the groups are organized in fixed positions because the groups (subjects, objects, permissions, and classes) are known and constant.

\section{Policy Developer Survey}

To better understand how policy analysts and developers currently work with SELinux policies, I conducted a survey of five people with SELinux policy analysis or development experience. Although not a large survey, this information can help us understand some of the current challenges faced in the real world and whether visualization tools such as V3SPA can address these challenges.

I recruited the survey respondents via e-mail from my professional network of people I knew to work with SELinux and/or SE for Android policies. The survey was sent to eight people, and five responded. Each respondent was offered a \$10 Starbucks gift card to participate in the survey. Each participant was asked six questions:

1. How many years of experience do you have with SELinux or SE for Android? (a) 0-1 years, (b) 1-2 years, (c) 2-3 years, (d) 3-4 years, (e) 4-5 years, (f) 5+ years.

2. Is most of your experience with (a) SE for Android, (b) SELinux, or (c) both about the same?

3. Is most of your experience with (a) creating policies, (b) analyzing policies, or (c) both about the same?

4. Which tools do you use to create policies?

5. Which tools do you use to analyze policies?

6. What is the most time consuming task of analyzing a policy?

The survey respondents had a range of experience working with SELinux or SE for Android policies, from 1-2 years of experience up to $5+$ years. Three of the respondents have worked primarily with SE for Android, one mostly with SELinux, one about the same amount of experience with both. Three of the respondents mostly have experience modifying policy, and the other two have about equal experience modifying and analyzing policies.

The survey respondents mostly use text editors to edit policy (nano, vim, and gedit). One respondent reported using audit2allow to generate new rules from audit logs, and two respondents reported using sepolicy-inject to add rules to a binary policy.

For analyzing policies, four respondents reported using grep, making it the most commonly mentioned tool. Respondents also use dmesg to monitor AVC denials, seinfo and sesearch (reported by two respondents each), and apol (reported by one respondent).

When asked what was the most time consuming task of analyzing a policy, two respondents indicated that searching across the many source files in a policy was the most time consuming. The other most time consuming tasks were understanding the structure of a policy, understanding policy variation across devices, understanding the behavior of the policy, and understanding the policy build tools and macros (each answer was given by one respondent).

From these responses, there is not much variety of tools used to edit policies. Mostly respondents use text editors. This makes sense, but indicates that policy developers might benefit from tools that provide code completion, refactoring support, syntax highlighting, or other tools found in modern programming environments.

There was more variation in the tools used to analyze SELinux policies. Searching appears to be a very common task, as indicated by the number of respondents that use grep and the one respondent that reported using sesearch. V3SPA has some support for searching in the policy explorer view, and both the policy explorer and the policy differ show users the full list of all subjects, objects, classes, and permissions, which can help users find a policy component if they are not sure of the name or where it occurs in the policy. The tools mentioned in the survey are primarily text based, which are not well suited to displaying large amounts of information that would be helpful when working with a large, complex dataset such as an SELinux security policy. This might explain why so many respondents reported that the most time consuming tasks when analyzing a policy have to do with searching and understanding the big picture of a policy. V3SPA was designed to address these issues by showing an entire policy at once, helping users identify a portion of the policy of interest, and narrowing their focus to identify specific details of the policy.

\section{V3SPA DESIGN}

V3SPA can load SELinux and SE for Android binary policies. V3SPA can also load SELinux policy source, assuming it is in the reference policy format, by using the Lobster [6] domain specific language. However, for brevity, this paper focuses on SELinux and 

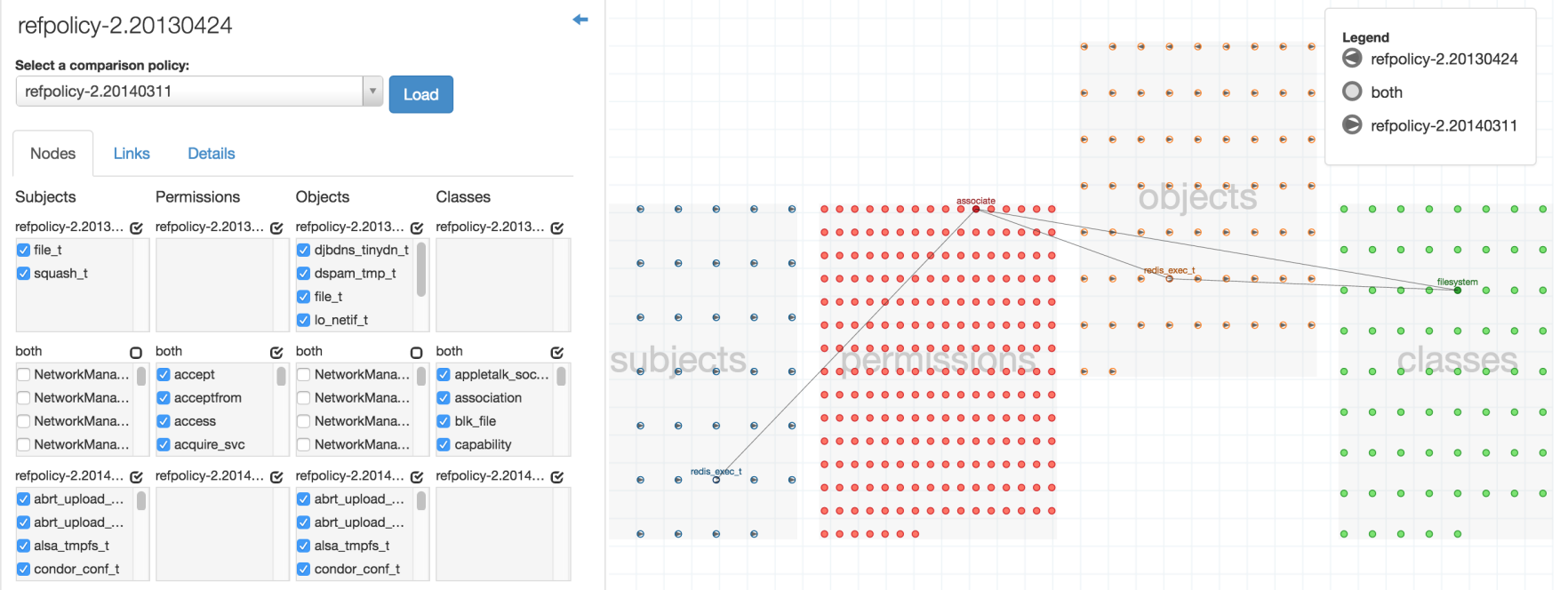

Figure 2: The policy differ in V3SPA. On the left, the editor pane shows controls for filtering the nodes in the policies, which are divided into subjects, permissions, objects, and classes, and further divided based on which policy contains those policy components. On the right, the nodes are broken into four groups. Hovering over the redis_log_t subject node shows all the subjects, permissions, and classes that co-occur in the same allow rules as the redis_log_t subject.

SE for Android binary policy support because V3SPA has similar features for analyzing policy binaries versus reference policy source.

V3SPA is primarily concerned with the type enforcement elements of SELinux security policies. In particular, V3SPA analyzes and visualizes the allow rules from security policies.

\subsection{System Architecture}

V3SPA is a web app with a browser-based frontend and a serverbased backend. The backend is a Python Tornado web server and a MongoDB database. The frontend and the backend communicate via REST API calls and websockets. When users load the V3SPA frontend in their browser, they can import an SELinux policy by uploading it in a zip archive. The zip archive can contain the policy binary, the policy source, or both. The frontend sends the policy to the backend, where the backend extracts the policy, uses sesearch from SETools version 4 to extract all allow rules from the policy binary, and then stores the text of the allow rules in the database.

When the frontend issues a call to the backend to retrieve the policy explorer graph or the policy differ graph, the backend calculates the graph in two steps. First, the backend parses the allow rule text to create an intermediate JSON representation of the allow rules that can be used to compute either the policy explorer graph or the policy differ graph. After parsing all the allow rules, the backend has created an array of intermediate JSON objects that are then cached in the database to avoid re-parsing the allow rules.

Second, the backend calculates the policy explorer graph, or the policy differ graph, depending on the request. If the graph is already stored in the database, the backend will send the previously calculated graph instead. If it is not already stored, it calculates the graph and caches it in the database before sending it to the frontend.

To calculate the policy explorer graph, the backend iterates over each intermediate JSON object created by the parser. The backend creates one node for each subject, and one node for each pair of objects and classes that both appear in the same rule (i.e. for the rule allow user_t bin_t : file \{read write\}; the backend would create one node called user $t$ and one called bin_t.file). The backend creates an edge linking a subject node and an object-class pair node if the subject has at least one permission on the object-class pair.

To calculate the policy differ graph for a single policy, the backend iterates over each intermediate JSON object and creates one node each for the subjects, objects, classes, and permissions in the policy. Edges indicate relationships between subjects, objects, permissions, and classes (e.g. which permissions a subject has). Edges connect subject nodes to permission nodes, permission nodes to object nodes and class nodes, and object nodes to class nodes if these policy components occur in the same allow rule. In the running example allow user_t bin_t : file \{read write ; the backend would create five nodes: one subject node user_t, one object node bin_t, one class node $\mathrm{f} i \mathrm{le}$, and two permission nodes read and write. The backend would also create edges from the user $t$ node to read and write nodes, from the read and write nodes to the bin_t and file nodes, and from the bin_t node to the file node. This graph structure mimics the way one might read the allow rule: The user_t domain has read and write permission objects of the bin_t type with the file class. This structure also allows users to see the relevant details (e.g. which objects or classes can a subject act on, what are those permissions, etc.) while keeping the number of edges in the graph to a minimum. When the frontend loads two policies, it then dynamically computes the differences between two policy graphs.

Graphs on the backend are stored in the $\mathrm{JSONH}^{6}$ format, which converts an array of JSON objects into a CSV-like array. This is much less verbose and consequently significantly fewer bytes in size than the uncompressed arrays of nodes and edges. When the frontend receives data from the backend, it then converts the JSONH graph back to JSON before further processing the graph.

\subsection{System Performance Optimizations}

The V3SPA system architecture includes several designs to improve performance. It is quite common to have SELinux policy graphs with tens of thousands of nodes and edges, but many off-the-shelf graph analysis tools have difficulty achieving good performance on graphs of this size. To aid other system designers in building performant graph analysis systems, both for cybersecurity-related and

${ }^{6}$ https://github.com/WebReflection/JSONH 
unrelated domains, I report on several design decisions that enable V3SPA performance to scale up to visualizing entire SELinux policy graphs on the order of tens of thousands of nodes and edges.

Caching. V3SPA makes extensive use of caching on the backend to avoid rerunning slow data processing algorithms. Specifically, V3SPA caches the parsed JSON version of the allow rules, and the policy explorer and policy differ graphs calculated from the JSON allow rules. Parsing the JSON and calculating the graphs can take tens of seconds, so caching the results in the database decreases future load time significantly.

Data compression. JSON is a verbose language, inflating the data size and causing long delays in transferring the data from the backend to the frontend. Most of V3SPA's data is stored as arrays of objects with identical structure, meaning that they repeat the same key names over and over. JSONH reduces the size of the data, as described above, so that key names are not repeated unnecessarily.

In the particular case of graphs, such as those in V3SPA, each edge references a source and target node. Instead of referencing the nodes by name (e.g. \{source: 'n1-name', target: 'n2-name'\}), the edges reference nodes by the nodes' indices in the node array (e.g. \{source: 1, target: 2 \} references the nodes at index 1 and index 2 in the node array). This is common because it is convenient for referencing nodes, but it also greatly reduces the storage requirements of edge objects, especially when combined with the JSONH format.

Finally, some node and edge data are needed for the visualization, but they can be computed on the frontend instead of the backend. For example, the policy differ needs to know the name of the policy that the subjects, objects, classes, and permissions come from. Instead of relying on the backend to attach this information to each object, the frontend can perform this action on load, reducing the amount of data sent from backend to frontend.

Combined, these techniques drastically reduce the amount of data sent from the backend to the frontend, often times from tens of megabytes down to a few hundred kilobytes. This drastically improves load times.

Canvas rendering. Many web-based visualizations are rendered using SVG, where roughly each visual element corresponds to one element in the browser's document object model (DOM). The downside is that browsers often experience considerable rendering performance loss when there are more than a few thousand DOM elements. Canvas is an alternative to SVG, where all visual elements are rendered as a single bitmap, which reduces the rendering burden on the browser. The policy explorer, which displays tens of thousands of nodes and edges, takes advantage of this to avoid suffering a frustrating rendering performance loss.

Data representation. The scheme for mapping policy components to graph nodes also has an impact on performance. The policy explorer represents pairs of objects and classes as an individual node, as opposed to having separate nodes for each. For the Tresys reference policy, this adds roughly 14,000 nodes in the graph compared to having separate nodes for each object and class, but it also reduces the number of edges in the policy explorer graph by about $33 \%$, or about 40,000 edges. This is useful for performance reasons, but it also reduces the density of the graph, making it more readable while still capturing important semantics.

\subsection{Policy Explorer}

On the frontend, when users select the policy explorer view, the frontend loads the policy explorer graph from the backend and renders the graph using the Sigma ${ }^{7}$ JavaScript library. The frontend uses a modified force-directed layout algorithm that is less accurate than Barnes-Hut force directed layout algorithms [16], but computes layouts faster. As described in Section 5.1, the policy explorer

${ }^{7}$ http://sigmajs.org/ shows a graph of subject nodes (dark blue) and object-class pair nodes (orange), where object-class pairs are created if the object and class both occur in the same allow rule. Edges connect subject and object-class nodes if the subject has one or more permissions on the object-class pair. This scheme is useful for understanding policy semantics, but it also improves reduces the number of edges, and therefore improves readability, as discussed in Section 5.2. Furthermore, users can click and drag nodes to change their positions in order to reduce occlusion and overlap. Figure 1 shows a screenshot of the policy explorer.

The policy explorer includes several controls for filtering the policy. These filters are divided into several tabs.

The Statistics tab has several range sliders to filter the nodes in the graph. Users can filter by node degree, node authority score [8], and node hub score [8]. Nodes with non-zero authority scores are object-class nodes, because they are the only nodes with incoming edges. Nodes with high authority scores are object-class nodes that have permissions on them by subject nodes that also have permissions on many other object-class nodes. Similarly, non-zero hub scores are associated with subjects, and nodes with high hub scores are subjects that have permissions on many object-class nodes. Therefore, authority and hub scores can be useful in identifying subjects and objects that may be over used in the policy. These filters can be effective for reducing clutter in the graph, improving readability, and identifying nodes to analyze. This is because node degrees in policy graphs tend to have a long tail distribution; most nodes have a small degree, but a few nodes have a large degree. Filtering nodes helps simplify the visualization.

The Access vector tab lists all of the subjects, objects, classes, and permissions found in the allow rules in the policy. Users can select specific components of access vector rules they wish to see or hide. For example, hiding specific subject nodes, or showing edges that represent specific permissions.

The Denials tab allows users to paste AVC (Access Vector Cache) denials and filter the policy to show only access vector components related to the AVC denial. Users can then see allow rules related to the denial, how allowing the denied functionality would affect the policy, and if there is a way to get around the denial.

The Always visible tab displays a list of nodes that are always visible, regardless of other filter settings. Users can type the name of a node and see a suggestion list of all nodes that contain that substring, and then select a node to add to the always visible list. Users can remove a node from the list by clicking the " $\mathrm{x}$ " delete icon next to a node name, or by clicking the "Clear all" button.

When users left click on a node, the Details tab shows all the allow rules associated with that node. This allows users to identify specific rules that may need to be modified or analyzed further.

Users can right click on a node to view a context menu about the node. This displays information about the node, including its degree, authority score, and hub score. Users can also see whether alternate versions of the node occur in the graph, i.e. if the user selected a subject node, the context menu also shows whether or not the subject also occurs as an object in the policy (or, conversely, if the node is an object, whether it also occurs as a subject). Users can then click the button to add those alternate nodes to the always visible list. Users can also add the selected node and/or its neighbors to the always visible list.

\subsection{Policy Differ}

In the policy differ view, users select which two policies they wish to see as a diff. The frontend then retrieves the policy differ graphs for both policies, and the frontend merges the two graphs by computing which nodes and edges are in only the first policy, which are only in the second policy, and which are in both policies.

The policy differ renders the merged graph of the two policies, where the nodes are divided into four groups: The subjects, the per- 
missions, the objects, and the classes. These groups are arranged from left to right in that order. This ordering mimics how someone might read an allow rule: This subject has this permission on these object types with this object class. The objects group is shifted vertically from the other groups to reduce the number of permissionto-class edges that cross the objects group.

The policy differ borrows visual language from other diff tools, where nodes with a left arrow are only in the first policy, nodes with a right arrow are only in the second policy, and nodes with a solid color are in both policies. This indicates to users whether a subject, permission, object, or class has been added or removed in a new version of the policy, or whether it was present in both policies. The nodes are also sorted so that nodes only in the first policy are displayed at the top, nodes only in the second policy are displayed at the bottom, and nodes in common between the two policies are in between. See the objects group in Figure 2 for an example.

When users hover the mouse cursor over a node, the policy differ highlights all other nodes representing policy components that co-occur in allow rules along with the hovered node, and connects them with edges. For example, if a user hovers over a subject node, then the policy differ highlights all of the object and class nodes that the subject has permissions on, as well as the corresponding permission nodes. Edges connect the highlighted nodes to make the relationships more clear.

Note that the nodes alone cannot capture all differences between two policies. For example, if we have the following two rules in version 1 of a policy:

allow user_t bin_t : file read;

allow user_t bin_t : dir write;

And in version 2 of the policy those two rules change to become allow user_t bin_t : file write;

allow user_t bin_t : dir read;

There are no new nodes, but the relationships between the nodes changed. Specifically, write is now connected to file, and read is now connected to dir. To see these differences in relationships, users can select to see only edges representing relationships only in the first policy, only in the second policy, or edges that are in both policies.

Users can click a node to lock the highlighted nodes and edges in place. This allows users to pan and zoom the visualization, or hover over other nodes to see their connected nodes and edges, while still seeing the clicked node and its connections. Clicking a node also displays all of the allow rules associated with that node and in the Details tab. Users can then see the rules, along with the name of the policy that contains each rule.

Users can also right click a node to get a context menu with several menu options to hide or show all connected nodes of each group (e.g. hide or show all permissions connected to a subject).

\section{Example Analyses}

The following examples illustrate how to use the policy explorer and the policy differ to examine SELinux security policies.

\subsection{Policy Explorer}

To demonstrate the policy explorer view, I load the binary policy from the Nexus 4 device (occam 5.1.1 build ID LMY48T).

At a high level, we see that there are a few dyads, i.e. a single subject node with permissions on a single object-class node. There also appear to be many object-class nodes that have permissions from only a few subject nodes. (See Figure 3a.) I quickly confirm this by adjusting the connections slider to hide nodes that have a high number of edges (connections higher than 1,100), which causes two subject nodes to disappear along with their edges. These two highly connected subject nodes are the unconfineddoma in node (connected to 1265 object-class nodes) and the init node (connected to 1142 object-class nodes). Many object-class nodes
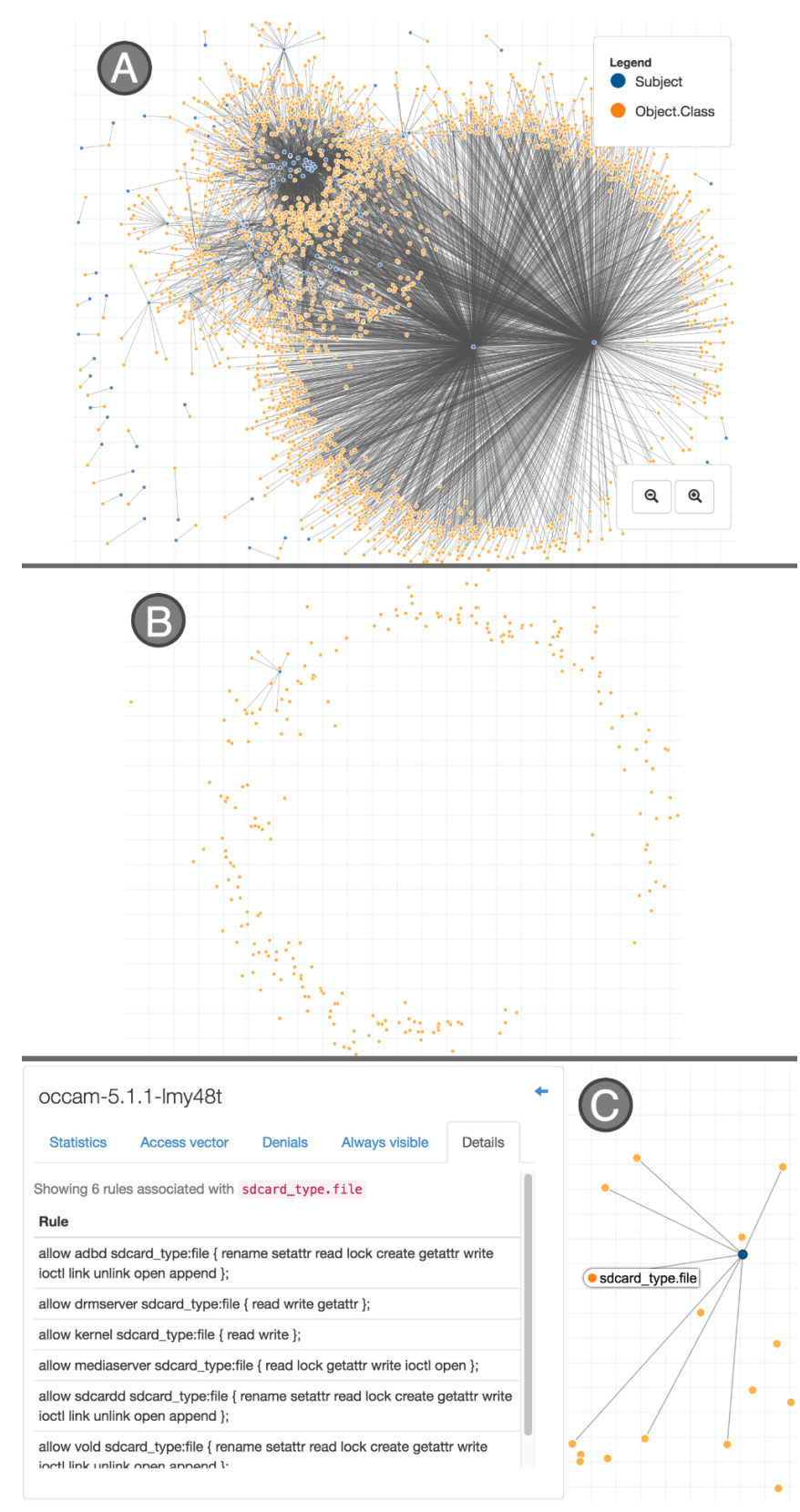

Figure 3: (a) Visualizing the overview of the occam 5.1.1 SE for Android policy. (b) Filtering using an AVC denial to show only the nodes and edges related to the denial. (c) Clicking the sdcard_type.file node shows the allow rules associated with that node. 


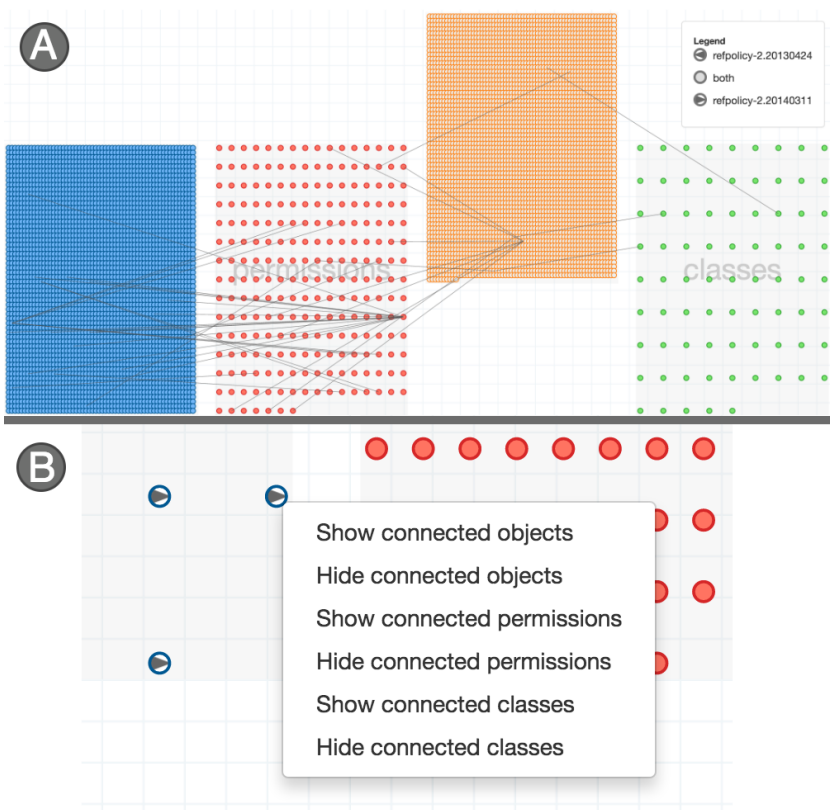

Figure 4: (a) Connections between policy components. These connections were removed after version 20130424. (b) A context menu gives users options for filtering connected nodes.

now become disconnected. Similarly, by hiding all nodes with fewer than 4 connections, most object-class nodes disappear.

If we tried to run this policy on our device and we got the following AVC denial, we could paste it into the Denials tab and see all of the related policy components.

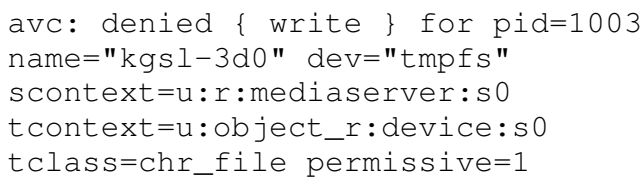

Doing so, we see the mediaserver subject node, and several object-class nodes that have either the device object or the chr_file class (see Figure 3b). The mediaserver subject node is connected to the video_device.chr_file, rpmsg_device.chr_file, camera_device.chr_file, tee_device.chr_file, gpu_device.chr_file, and audio_device.chr_file nodes via the write permission.

The user could choose to check if the mediaserver has permissions on a particular object-class pair, such as the sdcard_type.file node. To do so, users can open the "Always visible" tab, and begin typing "sdcard_type" in the input box. An autocomplete list of suggested node names appears, and the user can select "sdcard_type.file" from the list. This node is then added to the list of nodes that are always visible, and the node appears in the visualization. It is connected to the mediaserver subject node, and the user can click on the sdcard_type.file node to see all of its allow rules in the "Details" tab (see Figure $3 \mathrm{c}$ ). There are six allow rules, and we see that one of them gives mediaserver write permission on the sdcard_type object and $\mathrm{fil}$ le class.

If the user decides to add rules to address the AVC denial, the user could modify the policy, build it, re-import it, and then use the policy differ to verify that the changes only affect the desired portions of the policy.

\subsection{Policy Differ}

In this example, I load two consecutive versions of the Tresys reference policy. First I load the 20130424 release, and then I load the 20140311 release to compute a diff between them.

When the policy differ initially loads, it defaults to showing only policy components that have been added or removed from one policy to the next. We see two subject nodes with left arrows, indicating that these are subjects from the 20130424 policy that have been removed in the 20140311 policy. Meanwhile, 46 subjects have been added to the 20140311 policy. Similarly, we see 6 objects that were removed in the 20140311 version, and 59 objects that were added. There are no added or removed permissions or classes.

In the "Nodes" tab, we can select to see all of the permissions and classes. By doing so, we can hover over a subject and object node and see the other policy components associated with it. For example, we can hover over the redis_log_t subject and see that it has the associate permission on itself and the filesystem class (see Figure 2). This was policy functionality that was added in the 20140311 version.

One usecase of the policy differ is to verify that policy changes do not accidentally introduce unexpected behavior. For example, we might wonder if the redis_log_t has any permissions on other objects. To examine this, we can right click on the redis_log_t subject and click the "Show connected objects" menu item (see Figure 4b). Two objects are added to the objects group: the tmp_t and tmpfs_t objects (see Figure 5). Both of these objects are in both policies, as indicated by the fact that they are rendered as a solid-colored node. This indicates the redis_log_t has the behavior that we would expect, and there is nothing out of the ordinary.

To see changes in relationships between subjects and objects that exist in both policies, we can show only the nodes in common to both policies, make the links always visible, and show only the links in the 20130424 policy (see Figure 4a). This reveals that there are a small number of relationships that have been removed in the 20140311 version.

\section{Conclusion}

This paper presents V3SPA, a tool for exploring, analyzing, and diffing SELinux and SE for Android security policies. V3SPA has two main visualizations: A policy explorer, and a policy differ. The policy explorer is designed to help people who work with SELinux policies understand their policy better, a task that our survey respondents say is time consuming with the current ecosystem of tools. The policy differ is a visualization that supports differential policy analysis by showing a visual diff of two versions of a policy.

In addition to the visualizations, this paper makes several other contributions. I discuss several system architecture design decisions that enable V3SPA to scale up and visualize SELinux policy graphs with tens of thousands of nodes and edges. I also present a survey of five SELinux and SE for Android policy developers and analysts. The results of the survey indicate a need for powerful tools like V3SPA to help analysts understand the big picture of the large, complex security policies found in SELinux and SE for Android.

One avenue for future work is to design a better grid layout for the policy differ. Currently, the groups of nodes get crowded when there are many nodes visible. The policy differ would benefit from an elastic layout that dynamically adjusted the size of each group to accommodate the number of nodes currently in view.

\section{ACKNOWLEDGEMENTS}

I thank the V3SPA team for their support and technical contributions: Jeff Karrels, Kristina Creque, Daniel Su, Christopher Wacek, and Matthew Oertle. I also thank Joe Tanen for feedback and discussion, and the survey respondents for their time and insight. 


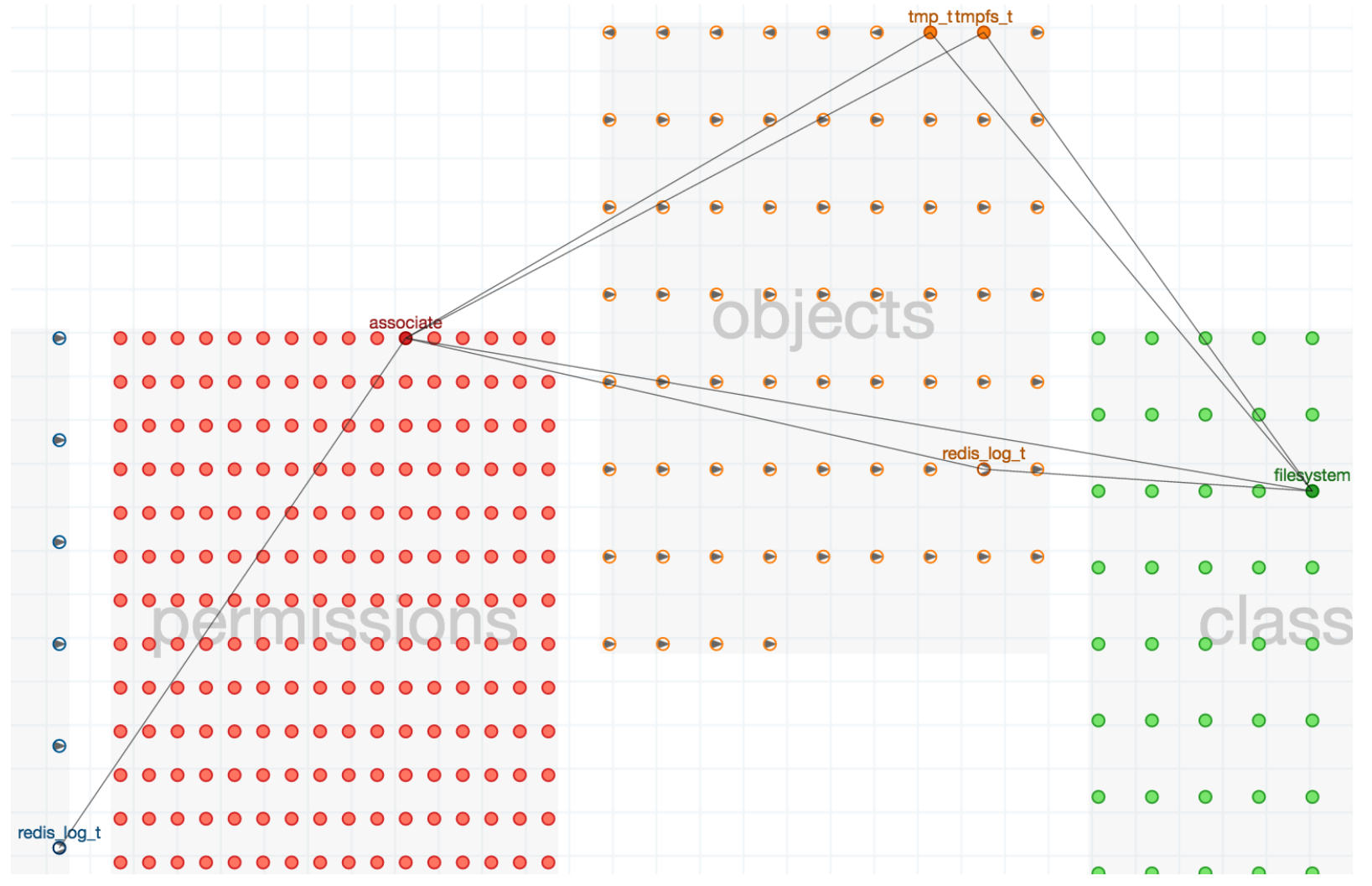

Figure 5: After showing objects connected to the redis_log_t subject, we see it has the associate permission on the tmp_t and tmpfs_t objects.

\section{REFERENCES}

[1] B. Bach, P. Dragicevic, D. Archambault, C. Hurter, and S. Carpendale. A Review of Temporal Data Visualizations Based on SpaceTime Cube Operations. In EuroVis, pages 23-41, 2014.

[2] M. Bastian, S. Heymann, and M. Jacomy. Gephi: An open source software for exploring and manipulating networks. 2009.

[3] H. Chen, N. Li, and Z. Mao. Analyzing and Comparing the Protection Quality of Security Enhanced Operating Systems. In NDSS, 2009.

[4] P. Clemente, B. Kaba, J. Rouzaud-Cornabas, M. Alexandre, and G. Aujay. SPTrack: Visual Analysis of Information Flows within SELinux Policies and Attack Logs. In Int. Conf. on Active Media Technology, pages 596-605, 2012.

[5] J. A. Guerra-Gomez, M. L. Pack, C. Plaisant, and B. Shneiderman. TreeVersity: Interactive Visualizations for Comparing Hierarchical Datasets. In Transportation Research Board, 2013.

[6] J. Hurd, M. Carlsson, S. Finne, B. Letner, J. Stanley, and P. White. Policy DSL: High-level Specifications of Information Flows for Security Policies. In HCSS, 2009.

[7] S. Kieffer, T. Dwyer, K. Marriott, and M. Wybrow. Hola: Human-like orthogonal network layout. TVCG, 22(1):349-358, Jan 2016.

[8] J. Kleinberg. Authoritative Sources in a Hyperlinked Environment. $J$. of the ACM, 46(5):604-632, 1999.

[9] P. Loscocco and S. Smalley. Integrating flexible support for security policies into the Linux operating system. In USENIX: FREENIX Track, pages 29-42, 2001.

[10] P. Loscocco, S. Smalley, P. Muckelbauer, R. Taylor, S. Turner, and J. Farrell. The inevitability of failure: The flawed assumption of security in modern computing environments. In NISSC, pages 303-314, 1998.

[11] P. Loscocco and S. D. Smalley. Meeting critical security objectives with Security-Enhanced Linux. In Ottawa Linux Symposium, pages 115-134, 2001.

[12] S. Marouf and M. Shehab. SEGrapher: Visualization-based SELinux policy analysis. In SafeConfig, pages 1-8, 2011.
[13] F. Mayer, D. Caplan, and K. MacMillan. SELinux by Example: Using Security Enhance Linux. Prentice Hall, Upper Saddle River, 2006.

[14] T. Munzner, F. Guimbretiere, S. Tasiran, L. Zhang, and Y. Zhou. TreeJuxtaposer: Scalable Tree Comparison using Focus+Context with Guaranteed Visibility. In SIGGRAPH, 2003.

[15] L. Pan and Q. Xu. Visualization Analysis of Multi-Domain Access Control Policy Integration Based on Tree-Maps and Semantic Substrates. Intelligent Information Management, 4(5):188-193, 2012.

[16] A. Quigley and P. Eades. FADE: Graph Drawing, Clustering, and Visual Abstraction. In Graph Drawing, pages 197-210, 2000.

[17] R. W. Reeder, L. Bauer, L. F. Cranor, M. K. Reiter, K. Bacon, K. How, and $\mathrm{H}$. Strong. Expandable grids for visualizing and authoring computer security policies. CHI, 1:1473-1482, 2008.

[18] E. M. Rodrigues, N. Milic-Frayling, M. Smith, B. Shneiderman, and D. Hansen. Group-in-a-box layout for multi-faceted analysis of communities. In PASSAT and SocialCom, pages 354-361, Oct 2011.

[19] S. Smalley and R. Craig. Security Enhanced (SE) Android: Bringing Flexible MAC to Android. In NDSS, pages 20-38, 2013.

[20] M. Smith, C. A., N. Milic-Frayling, B. Shneiderman, E. Mendes Rodrigues, J. Leskovec, and C. Dunne. NodeXL: a free and open network overview, discovery and exploration add-in for Excel 2007/2010/2013/2016. 2010.

[21] R. Wang, W. Enck, D. Reeves, X. Zhang, D. Xu, W. Zhou, and A. M. Azab. EASEAndroid: Automatic Policy Analysis and Refinement for Security Enhanced Android via Large-Scale Semi-Supervised Learning. In USENIX, pages 351-366, 2015.

[22] W. Xu, M. Shehab, and G. J. Ahn. Visualization-based policy analysis for SELinux: Framework and user study. Int. J. of Information Security, 12(3):155-171, 2013.

[23] W. Xu, M. Shehab, and G.-J. J. Ahn. Visualization based policy analysis: case study in SELinux. SACMAT, pages 165-174, 2008.

[24] V. Yoghourdjian, T. Dwyer, G. Gange, S. Kieffer, K. Klein, and K. Marriott. High-quality ultra-compact grid layout of grouped networks. TVCG, 22(1):339-348, Jan 2016. 